\title{
Correction to: Off-label-dosing of non-vitamin K-dependent oral antagonists in AF patients before and after stroke: results of the prospective multicenter Berlin Atrial Fibrillation Registry
}

\author{
Serdar Tütüncü ${ }^{1}$. Manuel Olma ${ }^{1,2}$. Claudia Kunze ${ }^{1}$ Joanna Dietzel ${ }^{1}$. Johannes Schurig ${ }^{1}$. \\ Cornelia Fiessler ${ }^{3}$. Carolin Malsch ${ }^{3,4}$. Tobias Eberhard Haas $^{3}$ - Boris Dimitrijeski ${ }^{5}$. Wolfram Doehner ${ }^{1,6}$. \\ Georg Hagemann ${ }^{7}$. Frank Hamilton ${ }^{8}$. Martin Honermann ${ }^{9}$. Gerhard Jan Jungehulsing ${ }^{10}$. Andreas Kauert $^{11}$. \\ Hans-Christian Koennecke ${ }^{12}$. Bruno-Marcel Mackert ${ }^{8} \cdot$ Darius Nabavi $^{5}$. Christian H. Nolte ${ }^{1,2,19,20}$. \\ Joschua Mirko Reis ${ }^{3} \cdot$ Ingo Schmehl $^{13} \cdot$ Paul Sparenberg $^{13} \cdot$ Robert Stingele $^{14} \cdot$ Enrico Völzke $^{15}$.

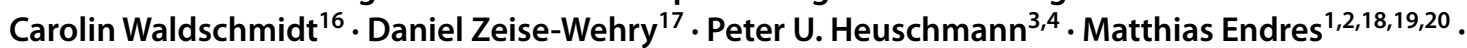 \\ Karl Georg Haeusler ${ }^{21}$
}

Published online: 27 November 2021

(c) The Author(s) 2021

\section{Correction to: Journal of Neurology} https://doi.org/10.1007/s00415-021-10866-2

The original version of this article unfortunately contained a mistake. Author name Matthias Endres was incorrectly written as Matthias Endress.

The correct spelling is "Matthias Endres".

\begin{abstract}
Open Access This article is licensed under a Creative Commons Attribution 4.0 International License, which permits use, sharing, adaptation, distribution and reproduction in any medium or format, as long as you give appropriate credit to the original author(s) and the source, provide a link to the Creative Commons licence, and indicate if changes were made. The images or other third party material in this article are included in the article's Creative Commons licence, unless indicated otherwise in a credit line to the material. If material is not included in the article's Creative Commons licence and your intended use is not permitted by statutory regulation or exceeds the permitted use, you will need to obtain permission directly from the copyright holder. To view a copy of this licence, visit http://creativecommons.org/licenses/by/4.0/.
\end{abstract}

The original article can be found online at https://doi.org/10.1007/ s00415-021-10866-2.

Karl Georg Haeusler

Haeusler_K@ukw.de

Extended author information available on the last page of the article 


\section{Authors and Affiliations}

\section{Serdar Tütüncü ${ }^{1} \cdot$ Manuel Olma $^{1,2} \cdot$ Claudia Kunze $^{1} \cdot$ Joanna Dietzel $^{1} \cdot$ Johannes Schurig $^{1}$. Cornelia Fiessler ${ }^{3}$. Carolin Malsch ${ }^{3,4}$ - Tobias Eberhard Haas ${ }^{3}$ - Boris Dimitrijeski ${ }^{5}$. Wolfram Doehner ${ }^{1,6}$. Georg Hagemann ${ }^{7}$. Frank Hamilton ${ }^{8} \cdot$ Martin Honermann $^{9} \cdot$ Gerhard Jan Jungehulsing $^{10} \cdot$ Andreas Kauert $^{11}$. Hans-Christian Koennecke ${ }^{12}$ - Bruno-Marcel Mackert ${ }^{8}$. Darius Nabavi ${ }^{5}$. Christian H. Nolte ${ }^{1,2,19,20}$. Joschua Mirko Reis ${ }^{3}$. Ingo Schmehl ${ }^{13} \cdot$ Paul Sparenberg $^{13} \cdot$ Robert Stingele $^{14} \cdot$ Enrico Völzke $^{15}$. Carolin Waldschmidt ${ }^{16}$. Daniel Zeise-Wehry ${ }^{17}$. Peter U. Heuschmann ${ }^{3,4}$. Matthias Endres ${ }^{1,2,18,19,20}$. Karl Georg Haeusler ${ }^{21}$}

1 Center for Stroke Research Berlin, CharitéUniversitätsmedizin Berlin, Berlin, Germany

2 Department of Neurology, Charité-Universitätsmedizin Berlin, Berlin, Germany

3 Institute of Clinical Epidemiology and Biometry, University Würzburg, Würzburg, Germany

4 Comprehensive Heart Failure Center, Clinical Trial Centre Würzburg, University of Würzburg, University Hospital Würzburg, Würzburg, Germany

5 Department of Neurology, Vivantes Klinikum Neukölln, Berlin, Germany

6 BCRT-Berlin Institute of Health Center for Regenerative Therapies, and Department of Cardiology (Virchow Klinikum), Charité-Universitätsmedizin Berlin, German Centre for Cardiovascular Research (DZHK), Partner Site Berlin, Berlin, Germany

7 Department of Neurology, Helios Klinik Berlin-Buch, Berlin, Germany

8 Department of Neurology, Vivantes Auguste-Viktoria-Klinikum, Berlin, Germany

9 Department of Neurology, Vivantes Klinikum Spandau, Berlin, Germany

10 Department of Neurology, Jüdisches Krankenhaus Berlin, Berlin, Germany
11 Department of Neurology, Evangelisches Krankenhaus Königin Elisabeth Herzberge, Berlin, Germany

12 Department of Neurology, Vivantes Klinikum im Friedrichshain, Berlin, Germany

13 Department of Neurology, BG Klinikum Unfallkrankenhaus Berlin, Berlin, Germany

14 Department of Neurology, German Red Cross Hospital Berlin Köpenick, Berlin, Germany

15 Department of Neurology, Schlosspark-Klinik Berlin, Berlin, Germany

16 Department of Neurology, Vivantes Humboldt-Klinikum, Berlin, Germany

17 Department of Neurology, Park-Klinik Weissensee, Berlin, Germany

18 German Center for Neurodegenerative Diseases (DZNE), Partner site Berlin, Berlin, Germany

19 German Center for Cardiovascular Diseases (DZHK), Partner site Berlin, Berlin, Germany

20 Berlin Institute of Health (BIH), Berlin, Germany

21 Department of Neurology, Universitätsklinikum Würzburg, Würzburg, Germany 